\title{
Hybrid Search Algorithms for P2P Media Streaming Distribution in Ad Hoc Networks*
}

\author{
Dong-hong Zuo, Xu Du**, and Zong-kai Yang \\ Dept. of Electronics and Information Engineering, Huazhong Univerisity of Science and \\ Technology, Wuhan, Hubei, 430074, China \\ \{sixizuo, duxu, zkyang\}@mail.hust.edu.cn
}

\begin{abstract}
Media streaming delivery in wireless ad hoc networks is challenging due to the stringent resource restrictions, potential high loss rate and the decentralized architecture. To support long and high-quality streams, one viable approach is that a media stream is partitioned into segments, and then the segments are replicated in a network and served in a peer-to-peer fashion, however, the searching strategy for segments is one key problem with the approach. This paper proposes a hybrid ant-inspired search algorithm (HASA) for P2P media streaming distribution in Ad Hoc networks. It takes the advantages of random walkers and ant-inspired algorithms for search in unstructured P2P networks, such as low transmitting latency and less redundant query messages. We quantify the performance of our scheme in terms of response time and network query messages for media streaming distribution. Simulation results show that it can effectively improve the Search Efficiency for P2P media streaming distribution in Ad Hoc networks.
\end{abstract}

Keywords: Ad Hoc networks, media streaming distribution, search algorithms, peer to peer.

\section{Introduction}

Media streaming distribution in wireless ad hoc networks is attractive and is also challenging due to stringent resource restrictions at the mobile hosts, dynamic network connectivity, and potentially high loss rate. To support media streaming applications with limited resources, one viable approach is: partitioning a media stream into segments and managing them in a peer-to-peer fashion [1, 2]. Unfortunately, this approach brings us challenges on searching for multiple segments and reassembling them in such unstructured P2P networks.

Flooding based search algorithms [1,2] are adopted due to the reliability and low latency. However, they incur large volume of unnecessary traffic to networks. Statistics based searching algorithms [3, 4] are proposed to avoid the large volume of redundant messages. But they introduce the partial coverage problem. In order to effectively reduce the redundant traffic and alleviate the partial coverage problem,

\footnotetext{
* Supported by Province Natural Science Foundation of Hu Bei under Grant No.2005ABA264.

** Corresponding auther.
} 
hybrid search algorithms [5] are proposed. But they are designed for the file sharing system and don't concern the correlation of continuous queries for media streaming segments.

We propose a hybrid ant-inspired search algorithm (HASA) for P2P media streaming distribution in Ad Hoc networks, coping with the P2P network churn and concerning the correlation of the searched media segments. It takes the advantages of $\mathrm{K}$ random walkers [4] and ant-inspired algorithms [6] for searching in unstructured media streaming distribution P2P networks.

\section{Description of the Hybrid Ant-Inspired Search Algorithm}

HASA uses the ant colony theorem [7] to collect and maintain the statistics information. At each peer $\mathrm{P}_{\mathrm{i}}$, pheromone trails are maintained in a pheromone table of size $\mathrm{m} \times \mathrm{n}$, where $\mathrm{m}$ is the number of the media segments and $\mathrm{n}$ is the number of peer Pi's outgoing links to neighbor peers $\mathrm{P}_{\mathrm{u}}$ where $\mathrm{u} \in\{1, \ldots, \mathrm{n}\}$.

\subsection{Overview of HASA}

Every peer in the network behaves as a media segments query originator peer, media segments providing peer, and/or intermediate peer. When a peer wants to find some media segment, a forwarding ant is generated. Originator peers and intermediate peers forward the forwarding ants according the forwarding rule. When the query is hit at one peer, a backward ant is generated, and is sent back following the reverse path of its corresponding forward ant. When ants move in the P2P network, they collect the paths' information and update the pheromone tables of peers in their paths according to the pheromone updating rule.

\subsection{Forwarding Rule}

Both intermediate and originator peers forward the ants in the same way. When a forward ant is received or generated, this ant will be forwarded to $\mathrm{K}$ neighbor peers except the incoming one. The next peer selection is done using the pheromone tables. If the amount of pheromones of all neighbors for the segment that the forward ant is searching for is $\tau_{\text {init }}$, then the forwarding ant will be forwarded to $\mathrm{K}$ randomly selected neighbor peers. Otherwise, if more than $\mathrm{K}$ links' pheromones for segment $\mathrm{s}$ are refreshed, the forwarding ant will be forwarded to these $K$ neighbors ( $K$ is far less than the number of neighbor peers.) that the amount of pheromone is more than the rest. If only $\mathrm{L}(\mathrm{L}<\mathrm{K})$ links' pheromones are updated, the forwarding ant will be forwarded to these $\mathrm{L}$ neighbors, and $\mathrm{M}(\mathrm{M} \leqq \mathrm{K}-\mathrm{L})$ neighbors those have more pheromone trails for segment $\mathrm{s}-1$. If $\mathrm{M}+\mathrm{L}<\mathrm{K}$, then other $\mathrm{K}-\mathrm{M}-\mathrm{L}$ neighbors will be randomly selected from the rest to be forwarded to.

\subsection{Pheromone Updating Rule}

In HASA the pheromone table is updated when peers receive either forward ants or backward ants. When a peer joins the media distribution network, we calculate the elapsed time into equal intervals. At startup, all peers' pheromone table entries are 
initialized with the same small value $\tau_{\text {init }}$. When receiving ants, peers update pheromone table using function (1). At the beginning of the nth interval, peers update pheromone table using function (2).

$$
\begin{gathered}
\tau_{\mathrm{s}, \mathrm{i}, \mathrm{j}}(\mathrm{n})=\left\{\begin{array}{cc}
\tau_{\mathrm{s}, \mathrm{i}, \mathrm{j}}(\mathrm{n})+\nabla \tau_{\mathrm{s}, \mathrm{i}, \mathrm{j}}(\mathrm{n}, \mathrm{am}) & \text { the incoming link } \\
\tau_{\mathrm{s}, \mathrm{i}, \mathrm{j}}(\mathrm{n}) & \text { other links } \\
\tau_{\mathrm{s}, \mathrm{i}, \mathrm{j}}(\mathrm{n})=\tau_{\mathrm{s}, \mathrm{i}, \mathrm{j}}(\mathrm{n}-1) \rho_{\mathrm{s}}
\end{array}\right.
\end{gathered}
$$

Where $\tau_{\mathrm{s}, \mathrm{i}, \mathrm{n}}(\mathrm{n})$ is the pheromone value for segment $\mathrm{s}$ corresponding to neighbor $\mathrm{j}$ at node $\mathrm{i}$ after passing $\mathrm{n}$ intervals. $\rho_{\mathrm{s}}$ is the evaporation parameter of pheromone trails for segment s. $\nabla \tau_{\mathrm{s}, \mathrm{i}, \mathrm{j}}\left(\mathrm{n}, \mathrm{a}_{\mathrm{m}}\right)$ is the pheromone left by the $\mathrm{mth}$ ants for segment $\mathrm{s}$ corresponding to neighbor $\mathrm{j}$ at node $\mathrm{i}$ in the $\mathrm{nth}$ interval. The amount of $\nabla \tau_{\mathrm{s}, \mathrm{i}, \mathrm{j}}\left(\mathrm{n}, \mathrm{a}_{\mathrm{m}}\right)$ are different at different peers which is left by the same ant. Example of such function is: $\nabla \tau_{\mathrm{s}, \mathrm{i}, \mathrm{j}}\left(\mathrm{n}, \mathrm{a}_{\mathrm{m}}\right)=\mathrm{w}_{\mathrm{p}} \mathrm{I}_{\mathrm{path,i}}+\mathrm{w}_{\mathrm{h}}\left(\mathrm{C}-\mathrm{h}_{\mathrm{s}, \mathrm{i}}\right)$, where $\mathrm{C}$ is a constant number, it should be larger than the longest hop for searching any segment $s$ in the network. $h_{s, i}$ is the hop that the ant has traveled from its generating peer to peer i. $\mathrm{I}_{\text {path }, I}$ is the path information from the originator or providing peer to the intermediate peer $i . \mathrm{w}_{\mathrm{p}}$ is the path information weight, and $\mathrm{w}_{\mathrm{h}}$ is the hop weight, they control the $\mathrm{w}_{p}+\mathrm{w}_{h}=1$. The path information is collected by ants.

\section{Performance Evaluation}

HASA is evaluated by simulating a peer-to-peer system with 1600 peers and by comparing its performance against that of the well-known $\mathrm{K}$ random walkers search[5] approaches. In this experiment, a long media stream is divided into 20 segments. Initially, all segments are stored in one peer in the network. Then streaming accesses are triggered at random points in the network. To model the popularity of the segments, a Zipf-like distribution is used. Each node has cache spaces for 3 segments. In the experiment described here, the parameter values were chosen as follows: $K=2$, $\rho_{\mathrm{s}}=0.2, \mathrm{w}_{p}=\mathrm{w}_{h}=0.5, \mathrm{TTL}=15$. To provide for comparison fairness, the TTL and $\mathrm{K}$ of $\mathrm{K}$ random walkers algorithm are set to 15 and 2 separately.

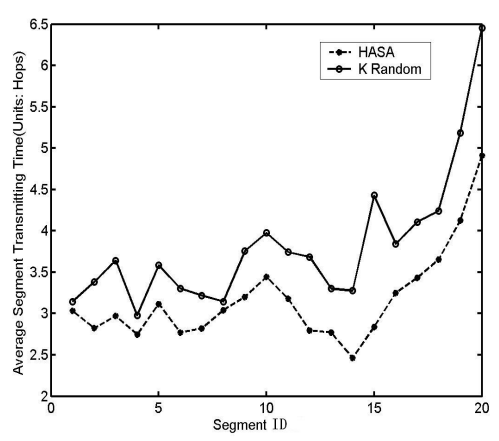

Fig. 1. The average segment transmitting delay

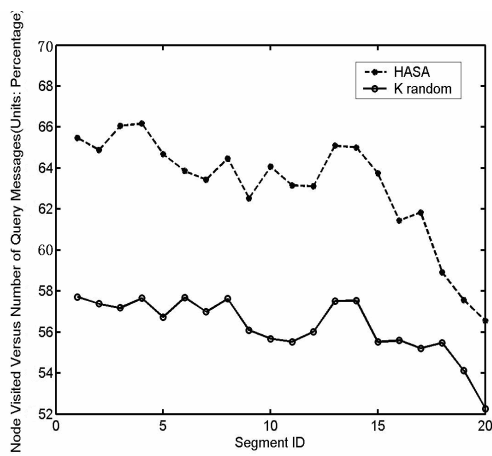

Fig. 2. The average ratio of nodes visited to number of query messages 
Fig.1 and Fig.2 show the performance comparison of the media transmitting latency and messages efficiency for $\mathrm{K}$ random walkers and the hybrid ant-inspired search algorithms. The simulation results show that the hybrid search algorithm is better than the $\mathrm{K}$ random walkers algorithm. HASA algorithm forward queries following the trails for latter generated searches, but $\mathrm{K}$ random walks still forwards queries randomly, so HASA algorithm can find the queried segment more quickly, and it can effectively avoid forwarding the queries to the useless peers.

\section{Conclusion}

HASA uses the K random walkers based search strategy for searching a segment at the beginning, when receiving queries and responses, peers update their pheromone tables immediately, then use the pheromone trails to forward $\mathrm{k}$ ants for searching segments at each intermediate peers or originator peers. It collects the advantages of $\mathrm{k}$ random walkers and ant-inspired search algorithms, it exploits the correlation of streaming access. It can reduce most redundant messages, but still keeps the low transmitting latency for media streaming distribution in wireless Ad Hoc networks.

\section{References}

1. Jin, S.: Replication of Partitioned Media Streams in Wireless Ad Hoc Networks. Proc. of the 12th ACM Int. Conf. on Multimedia. ACM press, New York (2004) 396 - 399

2. Shahram, G., Bhaskar, K. and Song, S.: Placement of Continuous Media in Wireless Peerto-Peer Networks. IEEE Trans Multimedia, Vol. 6, No. 2. IEEE Computer Society, New Jersey (2004) 335-342

3. Yang, B. and Garcia-Molina, H.: Improving search in peer-to-peer networks. Proc. of the 22nd Int. Conf. on Distributed Computing Systems. IEEE Computer Society, Washington (2002) 5-14

4. Qin, L., Cao, P., Edith, C., Li, K. and Scott, S.: Search and Replication in Unstructured Peer-to-Peer Networks. Proc. of the 16th Int. Conf. on Supercomputing. ACM Press, New York (2002) 84-95

5. Gkantsidis, C., Mihail, M. and Saberi, A.: Hybrid search schemes for unstructured peer-topeer networks. Proc. of IEEE INFOCOM 2005. IEEE Computer Society, New York (2005) 1526-1537

6. Yang, K.H., Wu, C.J., Ho, J.M.: AntSearch: An ant search algorithm in unstructured peerto-peer networks. IEICE Trans Commun, Vol. E89-B, No. 9. Oxford University Press, Bunkyo,Tokyo (2006) 2300-2308 